“Application of SeaWinds Scatterometer and TMI-SSM/I Rain Rates to Hurricane Analysis and Forecasting"

by R. Atlas (910), A. Hou (900.3) and O. Reale (910)

Popular summary

Results provided by two different assimilation methodologies involving data from passive and active space-borne microwave instruments are presented. The impact of the precipitation estimates produced by the TRMM Microwave Imager (TMI) and Special Sensor Microwave/Imager (SSM/I) in a previously developed 1D variational continuous assimilation algorithm for assimilating tropical rainfall is shown on two hurricane cases. Results on the impact of the SeaWinds scatterometer on the intensity and track forecast of a mid-Atlantic hurricane are also presented. This work is the outcome of a collaborative effort between NASA and NOAA and indicates the substantial improvement in tropical cyclone forecasting that can result from the assimilation of space-based data in global atmospheric models. 


\title{
Application of SeaWinds Scatterometer and TMI-SSM/I Rain Rates to Hurricane Analysis and Forecasting
}

\author{
Robert Atlas $^{\mathrm{a}, *}$ Arthur Y. Hou ${ }^{\mathrm{b}}$ Oreste Reale ${ }^{\mathrm{a}, 1}$ \\ ${ }^{a}$ Laboratory for Atmospheres, NASA Goddard Space Flight Center, Greenbelt, MD \\ 20771 \\ ${ }^{\mathrm{b}}$ Global Modeling and Assimilation Office, NASA Goddard Space Flight Center, \\ Greenbelt, $M D 20771$
}

\begin{abstract}
Results provided by two different assimilation methodologies involving data from passive and active space-borne microwave instruments are presented. The impact of the precipitation estimates produced by the TRMM Microwave Imager (TMI) and Special Sensor Microwave/Imager (SSM/I) in a previously developed 1D variational continuous assimilation algorithm for assimilating tropical rainfall is shown on two hurricane cases. Results on the impact of the SeaWinds scatterometer on the intensity and track forecast of a mid-Atlantic hurricane are also presented. This work is the outcome of a collaborative effort between NASA and NOAA and indicates the substantial improvement in tropical cyclone forecasting that can result from the assimilation of space-based data in global atmospheric models.
\end{abstract}

Key words: scatterometer, TMI-SSM/I, hurricane prediction, microwave remote sensing applications 


\section{Introduction}

Tropical cyclones are among the most devastating events in nature. However, as a result of improved computer models and remote sensing techniques, their impact on life loss have been gradually reduced. In this paper, we discuss results obtained by assimilating passive and active microwave remote sensing products in global atmospheric models with particular emphasis on tropical cyclones.

There are several intrinsic difficulties in the numerical forecasts of tropical cyclones. The first is the scale, which is relatively small for the current resolution of global models. The resolution needed to resolve the fine-structure of the tropical cyclone circulation would be of the order of $10-20 \mathrm{~km}$ or higher, whereas current global models used for operational weather forecasting operate at a resolution of $50-100 \mathrm{~km}$. As a consequence, the vortices represented in global models are too weak, their scale is too large, and their fine structure is not properly represented (Kurihara et al., 1990). The second problem is the position. Very often the location of the tropical cyclone-like signature in a global model could be misplaced of one or two gridpoints. This initial error, considered the scale involved, is quite large but can be further amplified in the forecast, leading to dramatically wrong tracks.

A traditional approach to reduce the errors in forecast track associated with the weakness and misplacement of hurricanes in the analyses is the bogusing

* Corresponding author. Tel.: +1-301-614-6140; fax: +1-301-614-6297 Email address: E-mail address: Robert.M.Atlas@nasa.gov (Robert Atlas).

1 Additional affiliation: University of Maryland, Baltimore County, Baltimore, Maryland 
technique (Lord, 1991), which consists of inserting an artificial vortex in the global model. The net result of bogusing is a stronger, smaller, more confined and better placed vortex, which may lead to improved forecasts. However, this approach has limitations: it can be successfully applied only to mature, highly symmetric hurricanes and problems related to how to specify a moisture field consistent with the imposed vortex are difficult to overcome ( $\mathrm{Pu}$ and Braun, 2001).

In this work, it is shown that two completely different methodologies which make use of microwave remotely sensed information can both act to 1) reduce the scale, increase the intensity and the realism of the representation of the tropical cyclone-like vortex in the analyses and 2) improve its position. Both techniques use quantitative satellite observations instead of artificially bogusing vortices. Therefore they are less prone to introduce artificial imbalances in the dynamics or in the physics of the model initial state. This work focuses on three cases of hurricanes: Bonnie (1998), Cindy (1999) and Floyd (1999).

Bonnie and Floyd are investigated to show the impact of the Variational Continuous Assimilation of the TRMM Microwave Imager (TMI) and Special Sensor Microwave/Imager (SSM/I), developed by Hou et al (2004) and based on Hou et al. (2000) and Hou et al. (2001). One important aspect of this methodology is that model errors are taken into account in the assimilation process. For this purpose, moisture and temperature tendencies are used as the control variables to compensate for model deficiencies, since model-predicted precipitation is diagnostically linked, via parametrization, to the rate of change in temperature and moisture. Therefore, observed surface rain rates are assimilated to estimate and correct for systematic errors in the temperature and/or moisture time-tendencies. The assimilation cycle is 6-hourly (Hou et al., 2004). 
The impact of SeaWinds Scatterometer on hurricane Cindy is studied according to the methodology described in Bloom et al (1999) and Atlas et al (1999). Scatterometer observations of the ocean surface wind speed and direction have been shown to improve the depiction and prediction of storms at sea (Atlas et al., 1999, 2001). These data are especially valuable where observations are otherwise sparse - mostly in the Southern Hemisphere and tropics, but also on occasion in the North Atlantic and North Pacific. The SeaWinds scatterometer on the QuikSCAT satellite was launched in June 1999 and it represented a dramatic departure in design from the other scatterometer instruments launched during the past decade (ERS-1,2 and NSCAT). More details on the SeaWinds instrument can be found in Atlas et al (2001) and Bloom et al. (1999). This work shows the influence of QuikSCAT data in data assimilation systems both from the NASA Data Assimilation Office (GEOS3) and from the National Centers for Environmental Prediction (the NCEP GDAS).

\section{TMI and SSM/I Experiments}

\subsection{Bonnie and Floyd assimilation experiments}

In this section, we show how the assimilation of tropical TMI and SSM/I surface rain rates over oceans affects the $1^{\circ}$ resolution analyses and forecast

of two distinct storms, Bonnie (1998) and Floyd (1999). Detailed information about the assimilation methodology in the GEOS-3 model can be found in Hou et al. (2004). The two storms were chosen because of their substantial differences. Bonnie was characterized by a remarkably asymmetric vortex, 
with winds much stronger on its northern side (Avila, 1998). It became a hurricane on 00 UTC 22 August 1998. Floyd was less asymmetric, and it became a hurricane on 12 UTC 10 September (Pasch et al., 1999). Hou et al. (2004) describe the various experiments which were performed. Here we focus on the improvement in the analyses that were obtained: on Bonnie at 12 UTC 21 August 1998 (12 hours before it reached hurricane strength) and on Floyd at 00 UTC 11 September 1999 (12 hours after reaching hurricane strength). At the times chosen, neither storm can be called a mature hurricane, and Bonnie is strongly asymmetric, so it would be impossible to apply the bogusing technique. Fig. 1 presents the meridional and zonal cross-sections of wind speed, temperature and relative vorticity across the center of Bonnie in the GEOS Analyses. We compare the control run (CNTRL) with the run in which rain râtes are assimilated (PRECIP). Both cross-sections show a better-defined warm core in the PRECIP case, and a substantial increase in low-level cyclonic vorticity. Particularly remarkable is the increase in asymmetry that can be seen on the meridional section (Fig. 1b). In fact, the wind is reduced to the south and increased to the north of the center, as a result of assimilating rain rates. This is in excellent agreement with available conventional observations (Avila, 1998). In Fig. 2 the same cross sections are shown for Floyd. Both meridional and zonal cross-sections show a substantial increase in low-level cyclonic vorticity, a better-defined warm core between 800 and $500 \mathrm{hPa}$, and an increase in the low-level wind. These improvements are not limited to this particular time but are generally consistent throughout the entire life-cycle of the two tropical cyclones. Simulations initialized with these improved analyses produce substantially more accurate forecast tracks (Hou et al., 2004). Since both Bonnie and Floyd did make landfall on the US mainland, the improvement in the forecast track obtained with this methodology has strong 
significance from the operational perspective and indicates potential for improved hurricane watches and warnings.

\section{SeaWinds Experiments}

\subsection{Discussion of results on a global scale}

As a result from a collaborative effort between NASA and NOAA, National Weather Service marine forecasters are using SeaWinds data to improve analyses, forecasts, and significant weather warnings for maritime interests. In this work we present some of the results emerged during this effort.

The strategy for assessing the impact of SeaWinds in NWP is described in Bloom et al (1999), and parallels the approach used in Atlas et al (1999) for the geophysical validation of NSCAT data. Two sets of early experiment were performed using data assimilation systems from both the NASA Data Assimilation Office (DAO) and the National Centers for Environmental Prediction (NCEP) using early data from SeaWinds. These early SeaWinds experiments

on GEOS-1, GEOS-2 and GEOS-3 were characterized by spin-up time of 10 days and assimilation window covering the period from 19 July to 19 September 1999. The following experiments were performed (each with 11 forecasts):

- CONTROL: all conventional data + TOVS + CTW

- QUIKSCAT: as the CONTROL + thinned QuikSCAT wind vectors

- SSM/I: as the CONTROL + SSM/I wind speed

- ERS-2: as the CONTROL + ERS-2 wind vectors

- SPEED: as the CONTROL + QuikSCAT wind speed 
The early SeaWinds experiment on the NCEP system were characterized by spin-up time of 10 days and assimilation window covering the period between 19 July to 12 September 1999. The following experiments were performed (each with 10 forecasts):

- CONTROL: all conventional data + TOVS + CTW

- QUIKSCAT: as the CONTROL + thinned QuikSCAT wind vectors (partial swath)

- NCEP-OP: as the CONTROL + SSM/I + ERS

- NCEP-OP-Q: as the NCEP-OP + thinned QuikSCAT wind vectors (partial swath)

- NCEP-OP-A: as the NCEP-OP + thinned QuikSCAT wind vectors (full swath)

- ADDITIONAL EXPERIMENTS: variable observation errors

Fig. 3 gives a detailed view of the influence that SeaWinds data can have on the positioning of a surface low pressure area in the analyses. The sea level analysis used in GEOS is multivariate; thus wind data can directly affect the analysis of sea level pressure. The circulation that can be inferred by the SeaWinds data indicates that the center of the cyclone in the control run is displaced of approximately $200 \mathrm{~km}$. The assimilation of SeaWinds can correct this error and locate the center of the cyclone in the assimilation run much closer to the center of the inferred circulation. This fact is particularly remarkable given the relative scarcity of conventional marine observations in the southern hemisphere. An improved representation of individual high-energy systems like cyclones is likely to improve the forecast.

A limited sample of 5-day forecasts were also made from initial conditions 
taken from assimilations made with and without SeaWinds data during the period 12-27 January, 2000. Fig. 4 shows the impact these data made on 500 $\mathrm{hPa}$ geopotential height anomaly correlations of forecast skill. There was a modest positive impact on forecast skill in the Northern Hemisphere, and a substantially larger improvement in forecast skill in the Southern Hemisphere. The reason of this difference is related with the density of conventional marine observations in the two hemispheres. In general it was observed that the $6 \mathrm{~h}$ wind vector difference and sea level pressure impacts are localized to the satellite swaths, but the assimilation system propagates the wind information considerably in the vertical. After $18 \mathrm{~h}$ from the initial time, the vector differences propagate also over all the oceans (not shown).

\subsection{Hurricane Cindy}

The impact of QuikSCAT data using the NCEP operational model is now discussed with reference to the case of hurricane Cindy. Cindy reached considerable strength (60 $\mathrm{ms}^{-1}$ and $942 \mathrm{hPa}$ on 28 August 1999) but did not make landfall and spent most of its lifetime over the eastern and central northern Atlantic before being absorbed by an extratropical low at 18 UTC 31 August (Guiney, 1999). Fig. 5 shows the impact of the assimilation of QuikSCAT on the vertical structure of Cindy, similar to what was shown for Bonnie and Floyd. Meridional and zonal cross-sections are presented at 00 UTC $29 \mathrm{Au}-$ gust 1999. Unlike Bonnie and Floyd, Cindy is a mature hurricane at this time. The impact of QuikSCAT is evident as a dramatic strengthening of the vortex. Cyclonic vorticity more than doubles in the lower levels, and a well-defined warm core appears in both the meridional and zonal cross-sections as a con- 
sequence of the QuikSCAT winds (Fig. 5b,d). The position of the storm is also improved by approximately $100 \mathrm{~km}$ relative to the "best track" (Guiney, 1999) provided by the National Hurricane Center (not shown). The consequence of the improved positioning and representation of Cindy leads to a substantially better forecast. Fig. 6 shows that the assimilation of SeaWinds data from QuikSCAT had an enormous beneficial influence on the amplitude and position of Cindy in a 60 hour forecast. The predicted center is approximately $10 \mathrm{hPa}$ deeper than the center predicted in the Control forecast and is much closer to the verifying analysis. Fig. 7 presents the magnitude and position errors for the Control (Operational) and QuikSCAT forecasts of Cindy. This experiment shows that the assimilation of QuikSCAT data results in a substantial reduction of both magnitude and displacement errors with respect to the control run. The $60 \mathrm{~h}$ forecast with QuikSCAT data is more accurate than the $24 \mathrm{~h}$ forecast without QuikSCAT data.

Hurricanes decaying to extratropical in the central or eastern Atlantic without making landfall have generally received little attention. Recent studies (Reale et al., 2001; Turato et al., 2004) however suggest that the extra-tropical remnants of eastern Atlantic hurricanes contribute to anomalous advection of moist tropical air masses towards Europe, occasionally leading to catastrophic floods over those regions. The results from this study are therefore also important from this operational perspective.

\section{Summary and conclusions}

In this study two different microwave remote sensing applications are shown to have a strong impact on the representation of hurricanes in global models 
and in their forecast.

In particular, we have shown that the technique of variational continuous assimilation of TMI and SSM/I rain rates developed by Hou et al. (2004) can have a significant positive impact on the representation of hurricanes. This is especially true at early stages of development, or in cases of strong asymmetry, when the bogusing technique could not be applied. In the future, more microwave rainfall data will become available possibly culminating with a constellation of 8 or more satellites providing 3-hourly measurements with the proposed Global Precipitation Measurement (GPM) mission. Therefore, the ability to assimilate rainfall information effectively even in presence of forecast model biases will be crucial.

SeaWinds data (like earlier observations provided by NSCAT or ERS) show unequivocal signatures of meteorological features including cyclones, fronts, anticyclones, easterly waves and other precursors of hurricanes and typhoons. Through collaborative efforts between NASA and NOAA, SeaWinds data are operationally used by National Weather Service marine forecasters. This results in substantial economic savings as well as the reduction of weather related loss of life at sea. The impact of SeaWinds on Numerical Weather Prediction models is on average modest but occasionally results in significant forecast improvements. In this study it is shown how the forecast track and intensity of a mid-Atlantic hurricane is remarkably improved.

The impact of SeaWinds data in Numerical Weather Prediction is currently limited by several factors. Among them are data redundancy (the same meteorological information may be detected by multiple components of the overall observing system), the accuracy of the current atmospheric model first guesses 
over oceans and the need of thinning the data. Occasional difficulties also arise with the quality control of these data. Finally, the error covariance models used in the data directly affect the weight given to the SeaWinds data with respect to a model first guess. Other limitations include the physical processes represented in the data that are not resolvable by the assimilation system. Improved impact of SeaWinds data would result from higher accuracy speeds and directions (such as would be achieved by improvements in the retrieval or directional ambiguity removal algorithms) and more accurate and sensitive quality control of the data.

However, in spite of these limitations, micro-wave remote sensing applications have a considerable impact on the prediction of hurricanes, because of the substantially improved representation of tropical cyclone-like vortices in the initial conditions of global models. The possibility of overcoming the inability of global models to produce realistic signatures of these vortices without inserting artificial forcings and without constraints dictated from the cyclone's maturity or symmetry, suggests an increasingly important role of microwave sensor products in operational weather forecasting.

\section{References}

R. Atlas, S.C. Bloom, R.N. Hoffman, E. Brin, J. Ardizzone, J. Terry, D. Bungato and J.C. Jusem, 1999. Geophysical validation of NSCAT winds using atmospheric data and analyses, J. Geophys. Res., 104(C5), 11405-11424.

Atlas, R., R.N. Hoffman, S.M. Leidner, J. Sienkiewicz, T.-W. Yu, S.C. Bloom, E. Brin, J. Ardizzone, J. Terry, D. Bungato, and J.C. Jusem, 2001. The effects of marine winds from scatterometer data on weather analysis and 
forecasting. Bull. Amer. Meteor. Soc. 82, 1965-1990, 2001.

Avila, L. A., 1998. Preliminary report. Hurricane Bonnie, 19-30 August 1998.

National Hurricane Center, 12 pp. [Available from Tropical Prediction Center, 11691 S.W. 17th Street, Miami, FL 33165-2149.]

S.C. Bloom, R. Atlas, E. Brin, J. Ardizzone, 1999. Validation of QuikSCAT SeaWinds Data at GLA. Reprint from the 13th Conference on Numerical Weather Prediction, Denver, CO., 11-14.

Guiney, J. L., 1999. Preliminary report. Hurricane Cindy, 19-31 August 1999. National Hurricane Center, 8 pp. [Available from Tropical Prediction Center, 11691 S.W. 17th Street, Miami, FL 33165-2149.]

Hou, A. Y., D. Ledvina, A. daSilva, S. Zhang, J. Joiner, R. Atlas, G. Huffman, and C. Kummerow, 2000. Assimilation of SSM/I-derived surface rainfall and total precipitable water for improving the GEOS analysis for climate studies. Mon. Wea. Rev., 128, 509-537.

Hou, A. Y., S. Zhang, A. daSilva, W. Olson, C. Kummerow, J. Simpson, 2001. Improving global analysis and short range forecast using rainfall and moisture observations derived from TRMM and SSM/I passive microwave sensors. Bull. Amer. Meteorol. Soc., 81, 659-679.

Hou, A. Y., S. Zhang, O. Reale, 2004. Variational Continuous Assimilation of TMI and SSM/I Rain Rates: Impact on GEOS-3 Hurricane Analyses and Forecasts. Mon. Wea. Rev., accepted.

Kurihara, Y. M., A. Bender, R. E. Tuleya, and R. Ross, 1990. Prediction experiments of Hurricane Gloria (1985) using a multiply nested movable mesh model. Mon. Wea. Rev., 118, 2185-2198.

Lord, S. J., 1991. A bogusing system for vortex circulations in the National Meteorological Center global forecast model. Preprints, 19th Conference on Hurricanes and Tropical Meteorology, Miami. Fl, Amer. Meteor. Soc., 328- 
330.

Pasch, R. J., T. Kimberlain and S. Stewart, 1999: Preliminary report. Hurricane Floyd, 7-17 September 1999. National Hurricane Center, 30 pp. [Available from Tropical Prediction Center, 11691 S.W. 17th Street, Miami, FL 33165-2149.]

Pu, Z. H., and S. Braun, 2001: Evaluation of bogus vortex techniques with four-dimensional variational data assimilation. Mon. Wea. Rev., 129, 20232039.

Reale, O., L. Feudale and B. Turato, 2001: Evaporative moisture sources during a sequence of floods in the Mediterranean region. Geoph. Res. Letters, $28,2085-2088$.

Turato, B., O. Reale and F. Siccardi, 2004. Water vapor sources of the October 2001 Piedmont filood. J. Hydrometeor., accepted. 


\section{List of Figures}

Fig. 1. Meridional (upper panels) and zonal (lower panels) cross-sections across the center of Bonnie at $12 \mathrm{UTC} 21$ August 1998 and at $65^{\circ} \mathrm{W}, 19^{\circ} \mathrm{N}$. Left panels indicate the control run, right panels the run in which rain rates are assimilated. Plotted are wind speed (shaded, $m s^{-1}$ ), relative vorticity (thick line, $s^{-1}$ ), temperature (thin line, ${ }^{o} C$ )

Fig. 2. Same as Fig. 1 but relative to Floyd at 00UTC 11 September 1999 at $60^{\circ} W, 21^{\circ} \mathrm{N}$.

Fig. 3. Sea Level Pressure analysis (hPa) at 12 UTC 23 August 1999 and QuikSCAT winds super-imposed, without QuikSCAT (top panel) and with QuikSCAT (bottom panel). The winds are in much improved agreement in the QuikSCAT case.

Fig. 4. Anomaly Correlation measures of forecast skill. $Q S C A T-P_{s}$ refers to a subset of SeaWinds data using a newer model function ("prime").

Fig. 5. Same as Fig. 1, but relative to Cindy at 00UTC 29 August 1999 and at $60^{\circ} \mathrm{W}, 31^{\circ} \mathrm{N}$. Right panels indicate the run in which QuikSCAT winds are assimilated.

Fig. 6. 60-h sea level pressure forecast (hPa) for 12UTC 31 August 1999 initialized at 00UTC 29 August 1999 without QuikSCAT (CNTRL, top panel) and with QuikSCAT (QSCAT, center panel) relative to Cindy. The shading indicate the difference QSCAT minus CNTRL. The panel below shows the NCEP validating analysis.

Fig. 7. Magnitude and displacement errors in the 60-hour prediction of the 
center pressure and center position by the NCEP system. 


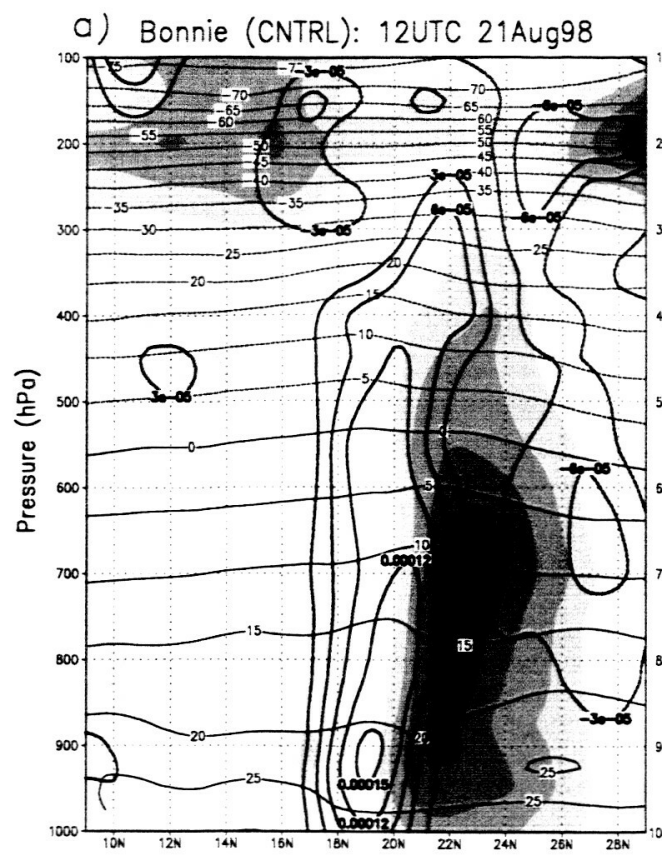

b) Bonnie (PRECIP): 12UTC 21 Aug98
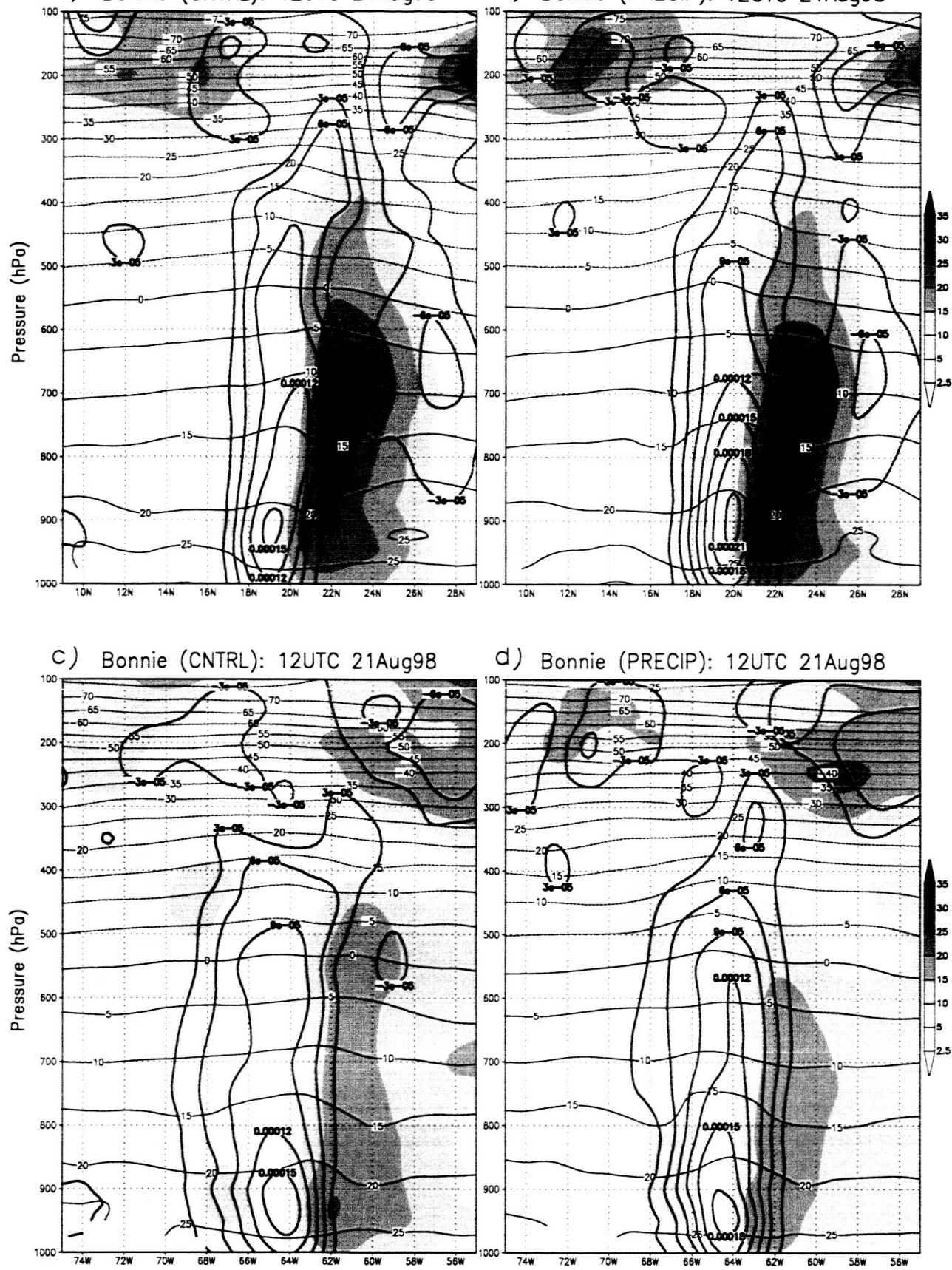

Fig. 1. 

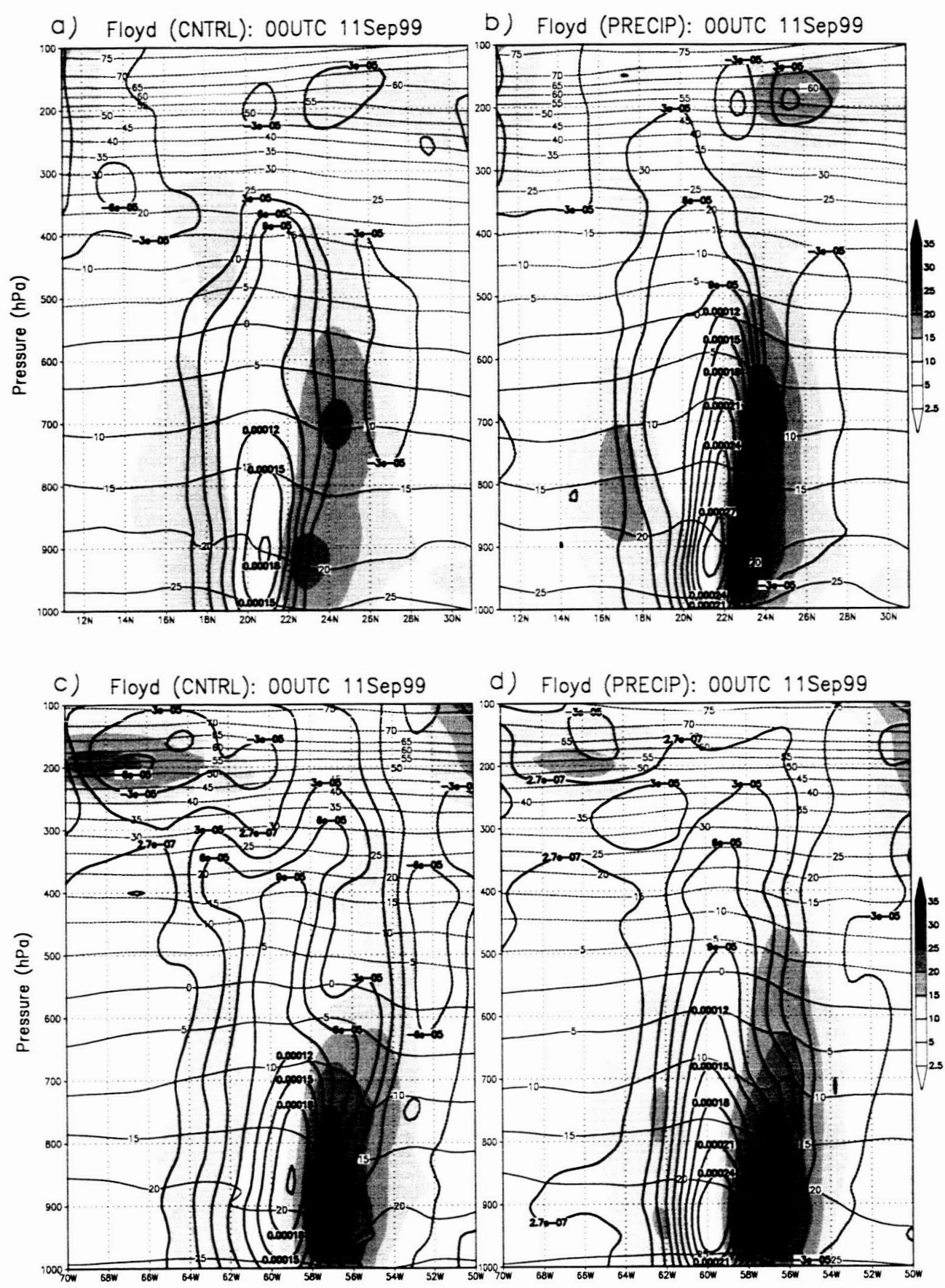

Fig. 2. 


\section{Impact of QuikSCAT Data on Surface Analysis}

Control

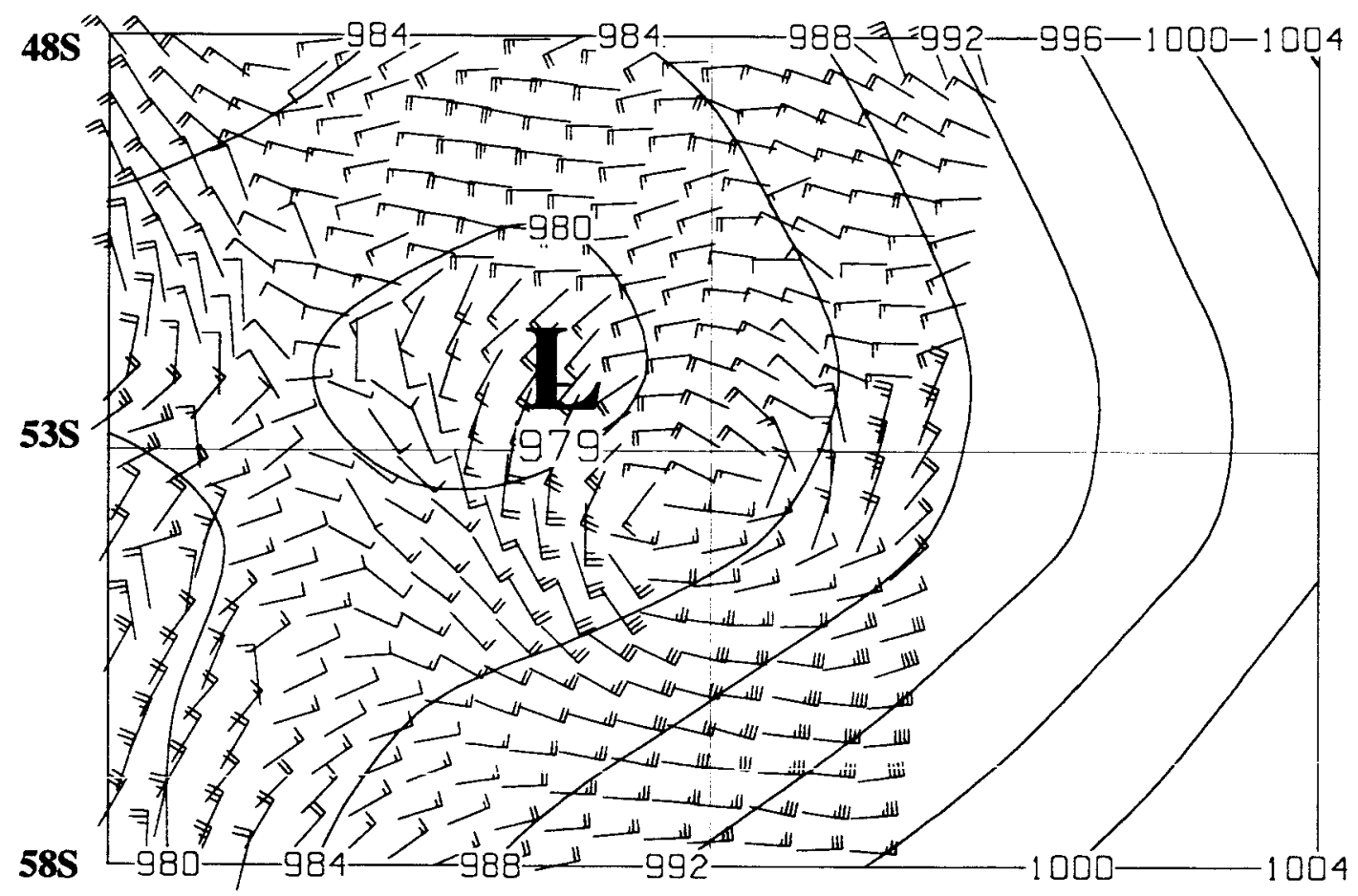

QuikSCAT

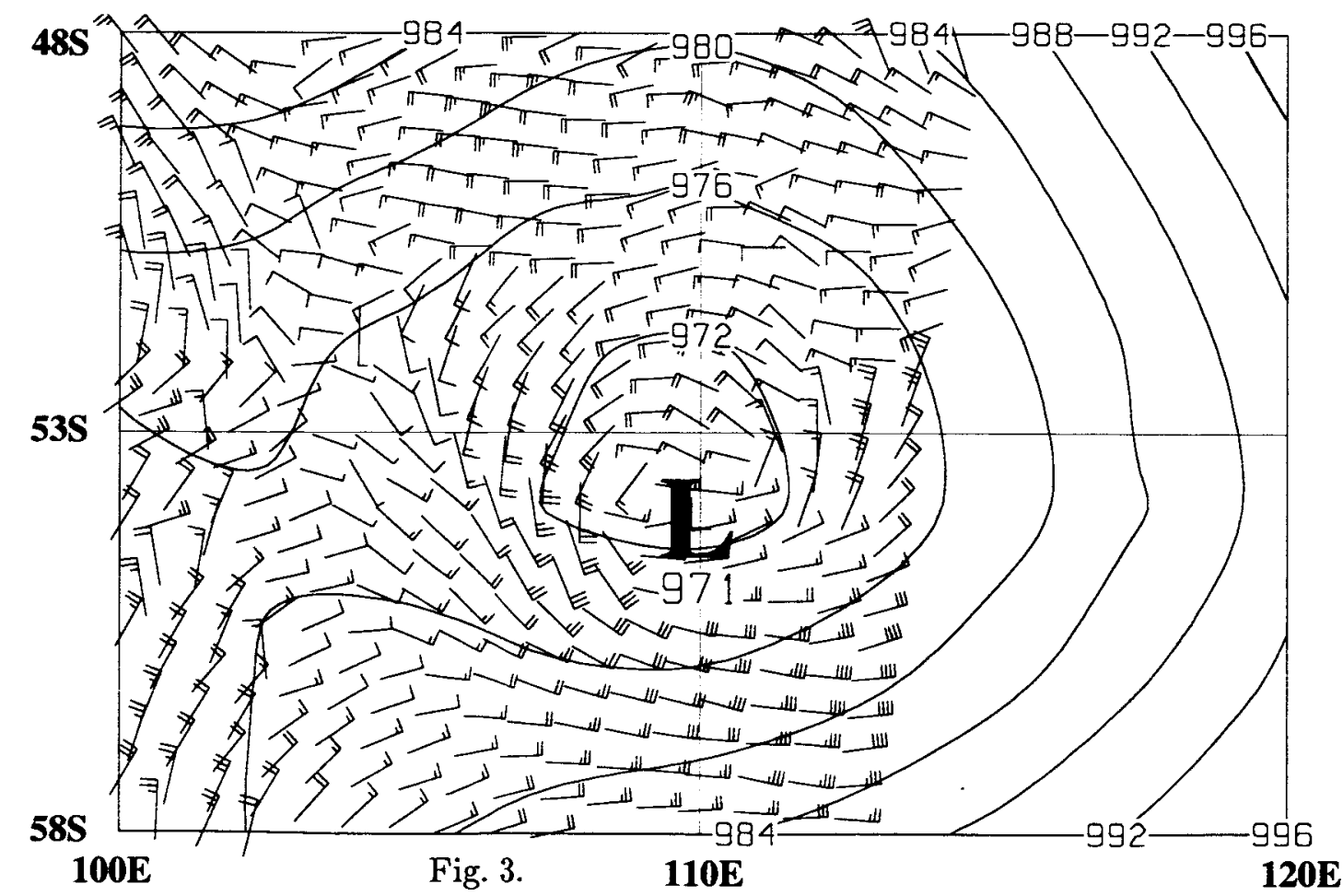




\section{Impact of QuikSCAT Data on GEOS-3 Forecasts}

September 2000

500 MB GEOPOTENTIAL HEIGHT - N. HEM. EXTRA TROPICS

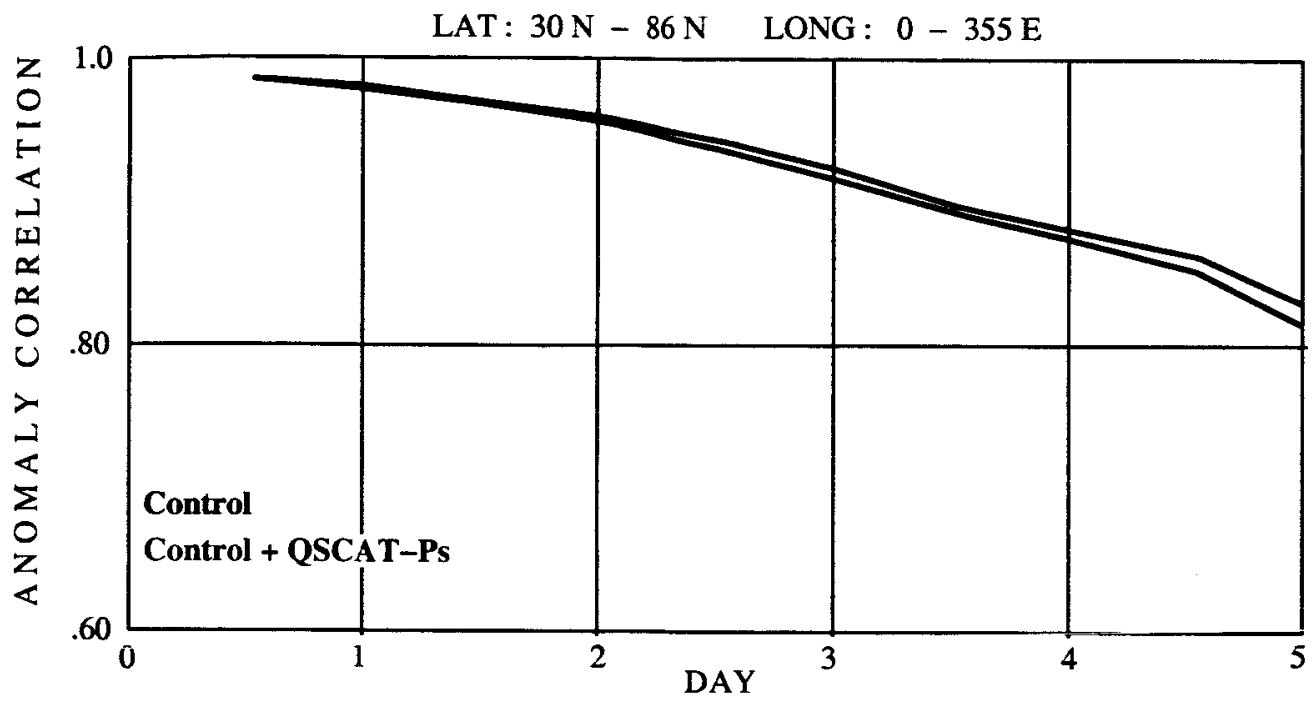

500 MB GEOPOTENTIAL HEIGHT - S. HEM. EXTRA TROPICS

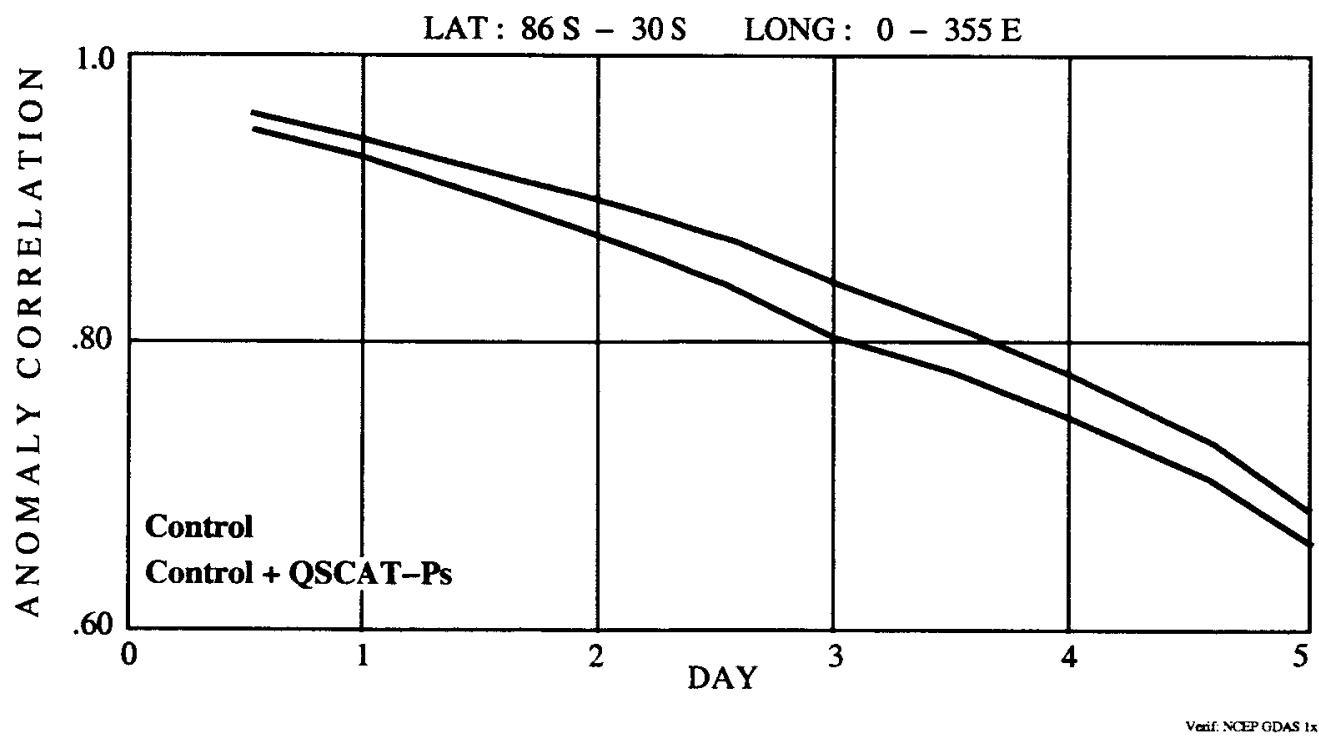

Fig. 4. 

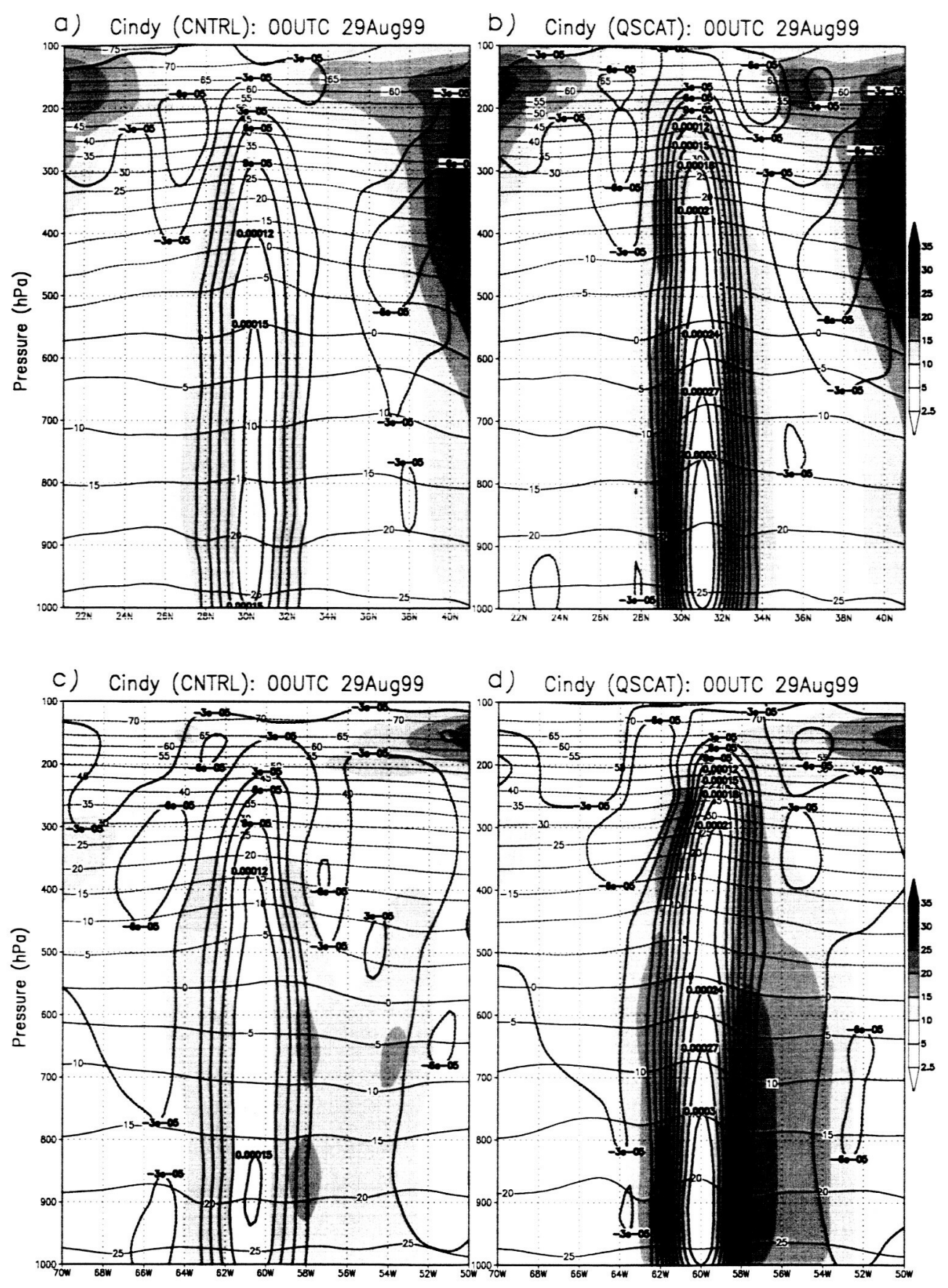

Fig. 5. 
FC 12z31Aug1999 IC 00z29Aug1999 (CNTRL)

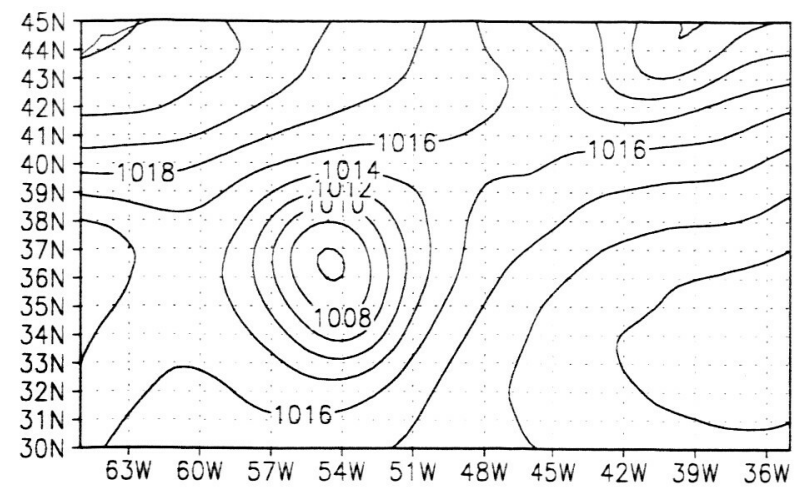

FC 12z31Aug1999 IC 00z29Aug1999 (QSCAT)
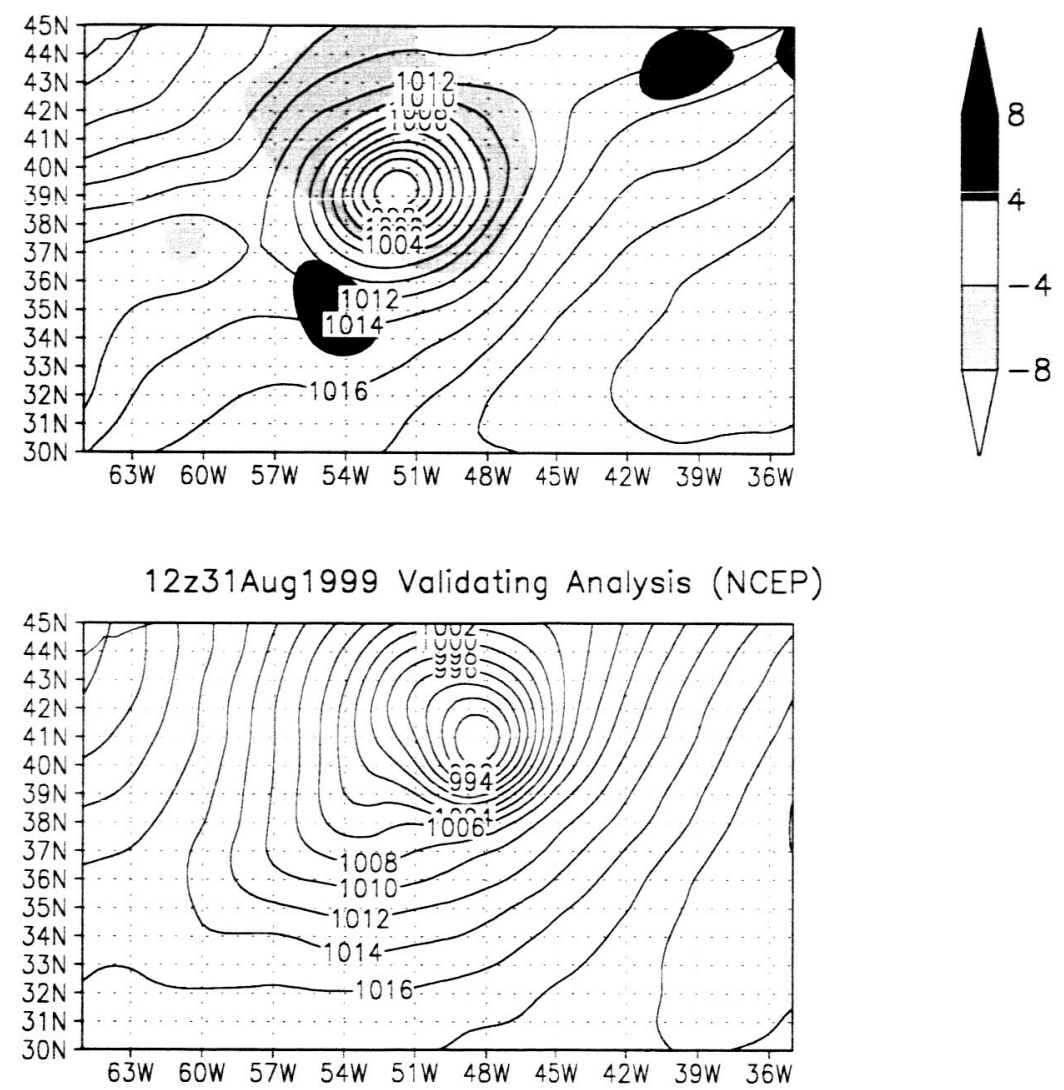

Fig. 6 . 


\section{Prediction of Hurricane Cindy using QuikScat Data}
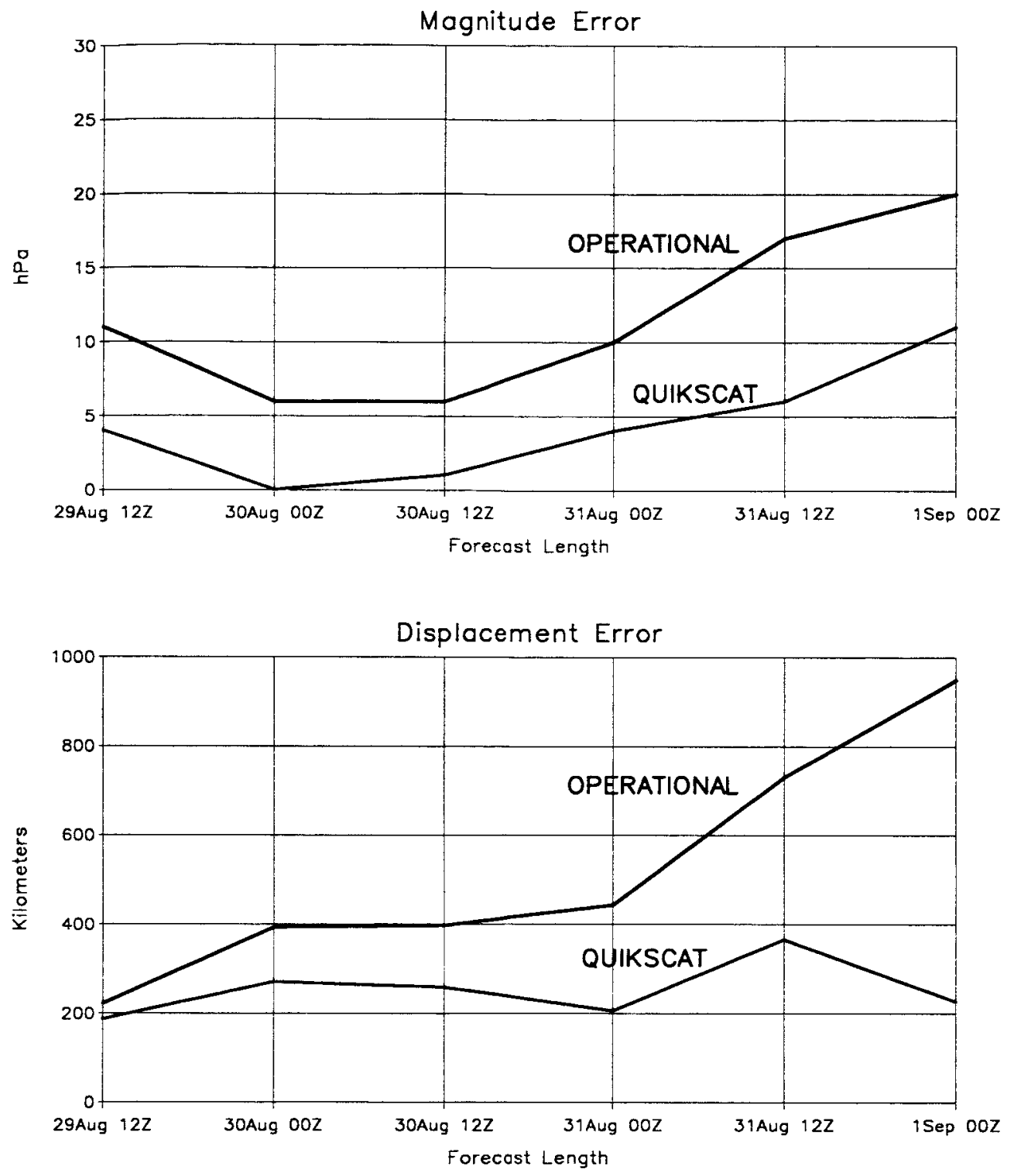

Fig. 7. 